\title{
Niveles de comprensión lectora en alumnos de secundaria. Una comparación por tópico
}

\author{
Levels of reading comprehension in high \\ school students. A comparison by topic
}

\author{
Yolanda Guevara Benítez ${ }^{1}$ \\ Karlena Cárdenas Espinoza ${ }^{2}$ \\ Verónica Reyes Pérez ${ }^{3}$ \\ Universidad Nacional Autónoma de México, México
}

\begin{abstract}
Resumen. Con base en los planteamientos de la psicología interconductual se desarrollaron y validaron dos pruebas para evaluar tres niveles de comprensión lectora de textos relacionados con dos tópicos: uno literario y uno de biología. El objetivo fue analizar el desempeño de estudiantes mexicanos de secundaria en comprensión lectora. Participaron 90 estudiantes de tres grupos escolares de primer grado, en una escuela pública. Se obtuvieron los puntajes de respuestas por nivel funcional. Los alumnos mostraron bajos porcentajes de respuestas correctas en ambas pruebas, siendo menores los relacionados con el tema de biología. Se ubicó a los alumnos en tres rangos de desempeño. Finalmente, se analizan las ventajas de evaluar la comprensión lectora por nivel funcional y por tópico.
\end{abstract}

Palabras clave. Comprensión lectora, niveles funcionales, tema literario, biología, educación secundaria.

Abstract. Under the theoretical basis of the interbehavioral psychology, were developed and validated two tests to assess reading comprehension of texts related with two topics: one literary and one of biology, in three functional levels. The aim was to analyze the performance of junior high school students, in reading comprehension. Participants were 90 students enrolled in the first grade of three school groups from a public school. The results show that students achieved low percentages of correct answers in both tests, in particular, their worst performance was related with the topic of biology. The results of the students were classified in three ranges of performance. The advantages of evaluating reading comprehension considering the functional levels of behavior and topic, are discussed.

Keywords. Reading comprehension, functional levels, literary theme, biology, secondary education.

\footnotetext{
${ }^{1}$ Yolanda Guevara Benítez. Facultad de Estudios Superiores Iztacala, Universidad Nacional Autónoma de México. Dirección postal: Avenida de los Barrios No. 1, Col. Los Reyes Iztacala. Tlalnepantla, Estado de México. C.P. 54090. E-mail: yolaguevara@hotmail.com

${ }^{2}$ Karlena Cárdenas Espinoza. Facultad de Estudios Superiores Acatlán, Universidad Nacional Autónoma de México. E-mail: karlenacardenas@hotmail.com ${ }^{3}$ Verónica Reyes Pérez. Facultad de Estudios Superiores Aragón. Universidad Nacional de México. E-mail: veroreyes68@yahoo.com.mx
} 


\section{Introducción}

Desde la perspectiva de la psicología interconductual, la conducta es definida como la interacción entre el individuo y el ambiente físico y social que lo rodea. La principal ventaja de esta perspectiva es que toma en consideración tanto al individuo como al contexto para explicar el desarrollo psicológico, lo que permite dar cuenta de diversos aspectos implicados en los avances cuantitativos y cualitativos del comportamiento humano.

Con base en dicho planteamiento, Ribes y López (1985) señalan que las interacciones pueden presentarse en formas cualitativamente diferentes, por lo que proponen una taxonomía que define cinco niveles funcionales de comportamiento, la cual da cuenta de diferentes grados de complejidad, dependiendo del grado de desligamiento de la conducta respecto de las situaciones presentes en tiempo y espacio. Estos autores explican que el desarrollo del comportamiento humano implica que el individuo va complejizando sus niveles de interacción, en conductas de diversa índole, siendo el lenguaje uno de los aspectos de mayor influencia en dicho desarrollo psicológico. Los individuos adquieren nuevos niveles de dominio lingüístico, asociados a un mayor grado de desligamiento de los eventos y objetos presentes en tiempo y espacio.

El uso del lenguaje en niveles complejos implica el dominio de convenciones lingüísticas variadas que permiten referir y ser referido a diversos aspectos de la realidad (en tiempos y espacios distintos a los presentes), pero para lograr ese grado de desarrollo lingüístico, el individuo ha de dominar primero niveles funcionales menos complejos.

Tales planteamientos interconductuales hacen posible estudiar no sólo el desarrollo del lenguaje oral, sino también el relativo al grado de dominio que se adquiere del lenguaje escrito, tarea que tradicionalmente corre a cargo del contexto escolar.

Autores como Guevara (2006) y Mares, Rueda, Rivas y Rocha (2009) recuperan la delimitación de los cinco niveles funcionales para describir habilidades de lecto-escritura implicadas en el contexto del salón de clases: Nivel 1 (contextual), el alumno responde a las características físicas de los eventos estímulo, sin alterarlos, únicamente ajustándose a los requerimientos del medio; participa en actividades que requieren un desligamiento mínimo del aquí y el ahora, ajustándose a los estímulos que el profesor le presenta; ejemplos de este nivel son escuchar el discurso didáctico del profesor, repetir ejemplos o definiciones mencionadas en clase previamente y copiar textos.

En el Nivel 2 (suplementario), el alumno modula su comportamiento, de modo que produce cambios en la situación interactiva actuando sobre su medio; se involucra en actividades instrumentales y prácticas, por ejemplo, formula preguntas o realiza ejercicios, sin modelo presente, pero siguiendo modelos previos. En el Nivel 3 (selector), el alumno debe elegir qué procedimiento seguirá o qué regla aplicará, de acuerdo con un criterio que puede variarde momento a momento; se comporta lingüísticamente o actúa sobre un objeto, mediado por la regla de correspondencia particular de una situación, lo que implica desligarse de una relación fija entre un objeto y otro, o entre una palabra y otra; selecciona entre varias opciones atendiendo aspectos convencionales previamente aprendidos y no presentes en tiempo y espacio; un ejemplo de este nivel es contestar preguntas seleccionando el producto lingüístico que responda a preguntas del tipo "qué", "quién”, "dónde”, “cuándo”, o ubicar el sujeto de la oración, verbos, sustantivos, entre otros (actividades típicas en las pruebas de comprensión lectora).

En el Nivel 4 (sustitutivo referencial), el alumno interactúa con objetos, personas, eventos, así como con sus propiedades, sin que estén presentes en la situación; el desligamiento es casi absoluto de la situación presente porque se refieren eventos pasados y se describen situaciones ausentes; un ejemplo es cuando los alumnos, a partir de los fenómenos o conceptos estudiados en clase (o leídos en un texto), refieren acontecimientos que les son familiares y los asocian con ellos. En el Nivel 5 (sustitutivo no referencial), el alumno interactúa con situaciones desligadas de los eventos concretos, con situaciones íntegramente convencionales, por ser de carácter simbólico; las interacciones lingüísticas entre los participantes van 
más allá de narrar, describir o referir eventos, objetos y personas, llegando a formular explicaciones con respecto a las relaciones entre eventos o juicios de valor; ejemplos de este nivel son argumentar, justificar, analizar y juzgar, con base en criterios de diversa índole, incluyendo razonamientos morales y científicos.

De esta manera, ser un lector competente implica la posibilidad funcional de responder efectivamente en tareas, contenidos y situaciones variadas, mostrando los diversos niveles funcionales de comportamiento (Arroyo et al., 2008; Irigoyen, Jiménez \& Acuña, 2007). La comprensión lectora con tales características es una condición indispensable para que el estudiante desarrolle habilidades relacionadas prácticamente con todas las materias escolares, debido a que la mayor parte de la instrucción educativa se basa en materiales escritos (Ibáñez \& Ribes, 2001). Las interacciones sustitutivas en el aula permiten que los alumnos sean capaces de aplicar, en contextos diferentes, los conceptos y operaciones aprendidos.

González, Barba y González (2010) explican que el bajo rendimiento académico en los estudiantes de secundaria tiene sus orígenes en deficiencias educativas escolares que vienen desde la primaria, donde se promueve la comprensión lectora casi exclusivamente en los cursos de Lengua y Literatura, a través de textos literarios, informativos y verbo-icónicos, dejando de lado el desarrollo de la comprensión de textos de índole científica. Al respecto, Pérez (2005) aclara que los textos literarios pueden incluir fragmentos descriptivos, narrativos o dialogados de cuentos, novelas, obras de teatro, u otros similares; los textos informativos incluyen noticias, recetas, cartas, instrucciones, folletos, prospectos, o temas de divulgación científica. Mientras que los textos verboicónicos incluyen rótulos, cómic, viñetas, anuncios, planos y otros parecidos. Agrega que estos tres tipos de textos se mencionan en el currículo establecido para los últimos grados de Educación Primaria.

Pérez (2005) señala que está ampliamente documentado que los textos que contienen una prosa narrativa literaria (junto con los otros dos tipos mencionados) son mucho más fáciles de comprender y retener que los que están conformados por una prosa expositivo-argumentativa, como la que se utiliza principalmente en los textos de ciencias naturales. Estos últimos están diseñados para informar y explicar relaciones entre eventos complejos que no siempre pueden asociarse con experiencias que el lector haya vivido, y la comprensión de los argumentos que manejan para explicar los fenómenos implica un mayor nivel de abstracción y de dominio lingüístico.

Considerando lo anterior, es importante llevar a cabo evaluaciones que den cuenta de los niveles funcionales de comprensión lectora que muestran los estudiantes de secundaria en lo relativo a textos de diversa índole. Las pruebas de comprensión lectora que estos estudiantes están habituados a responder (según constatan autores como Arroyo, Morales, Silva, Canales \& Carpio, 2011; Backhoff, Sánchez, Peón \& Andrade, 2010; Bazán, Castañeda, Macotela \& Valenzuela, 2004; Irigoyen, Acuña \& Jiménez, 2011; Llorens et al., 2011), la mayoría de las veces, tienen un sistema de calificación que se basa en la selección de las respuestas correctas, dentro de un conjunto de opciones (pruebas de opción múltiple). Ello implica que pueden ubicarse en un nivel 3 (selector) de comportamiento, de acuerdo con las definiciones antes expuestas. Tal característica de los instrumentos impide contar con datos relacionados con diferentes niveles de desempeño en la comprensión lectora de los alumnos, así como evaluar si dichos niveles de comprensión varían de un tópico a otro.

El objetivo del presente estudio fue analizar las características del desempeño de estudiantes mexicanos de secundaria, en la comprensión lectora de textos con dos tópicos distintos -un tema literario y otro de biología-, a través de sus respuestas en pruebas diseñadas para identificar los niveles funcionales de comportamiento lector de mayor complejidad (selector, sustitutivo referencial y sustitutivo no referencial).

Con base en los planteamientos expuestos, se formuló como hipótesis que los alumnos mostrarían mejores desempeños en la prueba basada en un tema literario, que en la relativa a un tema de biología. 


\section{Método}

Participantes. Noventa estudiantes, 53\% de sexo femenino y $47 \%$ de sexo masculino, con edades entre 12 y 14 años, y una media de edad de 12 años 9 meses; inscritos en tres grupos escolares del turno matutino de primer grado de secundaria, en una escuela pública de la Ciudad de México.

Instrumentos y materiales. Con base en los planteamientos interconductuales expuestos, particularmente en las definiciones de Guevara (2006) y Mares et al. (2009), se desarrolló un instrumento para medir la comprensión lectora en los tres niveles de mayor complejidad funcional (selector, sustitutivo referencial y sustitutivo no referencial), conformado por una prueba relacionada con un tema literario un cuento corto titulado "Cinco dracmas" que contiene 893 palabras; y otra prueba relativa a un tópico de biología, un texto denominado "La biodiversidad: resultado de la evolución” con 1032 palabras, que forma parte de la primera unidad de un libro de biología para primer grado de secundaria. Cada prueba incluye, además del texto, nueve reactivos dirigidos a identificar respuestas que denotan interacciones en los tres niveles funcionales (tres preguntas por nivel). Cada reactivo cuenta con un criterio de respuesta, para ser considerada como correcta o incorrecta, con base en los criterios que se derivan de la definición de cada nivel funcional.

El tema literario narra la historia de un niño de nombre Ian a quien uno de sus compañeros hace víctima de acoso escolar. A continuación se presentan algunos ejemplos de las preguntas de comprensión: 1) para evaluar el nivel selector se le presentaron al alumno preguntas con imágenes que debía mirar para responder, una de las preguntas fue "Menciona tres características físicas en las que el niño que aparece en esta imagen se parece a Ian" (la descripción del niño se encuentra en el texto leído); 2) para evaluar el nivel sustitutivo referencial se le pidió que narrara algún caso que conociera y que fuera similar al de Ian; 3) para el sustitutivo no referencial se le pidió que propusiera acciones para evitar que, en las escuelas, se presente el problema narrado (acoso escolar).

Por otro lado, ejemplos de los reactivos del tópico de biología son los siguientes: 1) para evaluar el nivel selector se le presentaron al alumno las preguntas con imágenes que debía mirar para responder, una de ellas fue "Menciona una característica que tienen en común las ranas y las moscas, que les ha permitido adaptarse a su ambiente" (la respuesta literal se encuentra en la lectura); 2) para el nivel sustitutivo referencial se le pidió que mencionara tres organismos pluricelulares distintos a los que señala el texto; 3) para evaluar el sustitutivo no referencial se le preguntó “¿Por qué crees que los osos polares y los zorros del ártico son peludos y blancos? Argumenta tu respuesta con lo que sabes del tema".

Todos los materiales del instrumento se sometieron a un proceso de validación por jueces expertos, quienes fungieron como tales fueron cinco profesores universitarios con experiencia de 10 años o más en psicología interconductual y en investigación educativa, para que emitieran sus opiniones en relación a: 1) pertinencia de la extensión y terminología del texto, de acuerdo al nivel escolar de alumnos de primer grado de secundaria; 2) pertinencia de las preguntas para identificar respuestas en cada uno de los niveles funcionales que se pretendía evaluar; 3) pertinencia de la respuesta considerada como correcta para cada pregunta, y 4) pertinencia de las ilustraciones que se incluyeron en el cuestionario de evaluación. Con base en las opiniones de los jueces se realizaron algunos ajustes para contar con una versión validada del instrumento.

Escenario. Las evaluaciones con los alumnos participantes se llevaron a cabo en un aula de 4 x 3 metros, bien iluminada y ventilada, designada por la dirección de la escuela.

Procedimiento. Se visitaron varias escuelas secundarias públicas y se expusieron a sus directores los objetivos y características del estudio, explicando que el interés era estudiar el nivel de comprensión lectora con que los alumnos ingresan a secundaria, por lo que era importante estudiar a los grupos escolares, tal como 
están conformados. El estudio se llevó a cabo en la escuela cuyas autoridades, profesores y padres permitieron el acceso y otorgaron su consentimiento informado. Se realizó una sesión informativa con cada uno de los tres grupos escolares de la escuela y se explicó a los alumnos que su participación en el estudio era voluntaria, que los resultados no afectarían su calificación en ninguna de las materias, y que los datos obtenidos serían confidenciales. Se aplicaron las evaluaciones a los alumnos que aceptaron participar.

La aplicación de las dos pruebas (una de cada tópico) se llevó a cabo, para cada uno de los grupos por separado, en sesiones de aproximadamente una hora de duración.

Calificación y confiabilidad. Con base en los criterios de definición de cada nivel funcional, se llevó a cabo la calificación de todas las pruebas aplicadas, por parte de dos evaluadores independientes. Para determinar el grado de coincidencia entre evaluadores se utilizó la fórmula: Acuerdos /Acuerdos + Desacuerdos $\times 100$. Las calificaciones fueron capturadas en una base de datos del programa SPSS Versión 15.0 para obtener promedios de respuestas correctas para cada prueba, en cada nivel funcional, así como en una base del programa Microsoft Office Excel 2007 para obtener porcentajes. Se aplicaron dos pruebas estadísticas a los datos de la muestra total, una prueba $t$ de Student y un ANOVA one way, con el propósito de conocer las diferencias entre las medias de ejecución de los alumnos, en la comprensión lectora de cada tópico (literario y de biología), así como en el promedio de ejecución mostrado en cada nivel funcional evaluado. Se consideró el porcentaje de respuestas correctas en el tema literario para ubicar a los alumnos en tres rangos de ejecución: Bajo (de 0 a 33.3\%), Medio (de 33.4 a $66.6 \%$ y Alto (66.7\% o más) y se compararon sus calificaciones en dicho tópico con las obtenidas en el tema de biología.

\section{Resultados}

Se obtuvo un índice de confiabilidad entre evaluadores de $93 \%$ en promedio para ambas pruebas, $94 \%$ en el caso del tema literario y $92 \%$ en el tópico de biología.
La media de calificación de la muestra de alumnos $(\mathrm{N}$ $=90)$ fue de 4.49 en la prueba sobre el tema literario y de 2.79 en la del tópico de biología, de un total de 9 puntos posibles de calificación por prueba; ello correspondió a ejecuciones de $50 \%$ y $31 \%$ de respuestas correctas, respectivamente. La prueba $\mathrm{t}$ para una sola muestra indicó diferencias estadísticamente significativas entre las calificaciones totales de la prueba del tópico literario $(M=4.50, D E=2.50), t(89)=-17.50, p<.001, d=$ 0.74 , respecto a los obtenidos en la prueba del tópico de biología $(M=2.79, D E=2.11), t(89)=-27.82, p<.001$, $d=0.17$.

Los porcentajes de respuestas correctas relacionadas con el tópico literario fueron de 55\% para el nivel selector, $54 \%$ para el sustitutivo referencial (SR) y $61 \%$ para el sustitutivo no referencial (SNR). En el caso del tema de biología, los porcentajes fueron de $51 \%, 42 \%$ y $44 \%$, respectivamente.

Con base en los puntajes obtenidos en el tema literario se localizaron 27 participantes ubicados en un rango Bajo de calificación, 44 en un rango Medio y 19 en un rango Alto. La figura 1 muestra los porcentajes promedio obtenidos por los alumnos ubicados en cada rango de calificación.

La Tabla 1 muestra los puntajes promedio obtenidos por los alumnos ubicados en el rango Bajo de calificación, así como las diferencias que mostraron entre ambos tópicos, por nivel funcional evaluado y en el puntaje total de las pruebas.

Al comparar el puntaje total de ambas pruebas, los participantes situados en el rango Bajo tuvieron menores ejecuciones en el tópico literario $(M=15.07)$ que en el de biología $(M=23.81)$. La comparación de los puntajes por nivel funcional indicó que el nivel selector fue el de mayor ejecución en los dos tópicos $(M=19.70$ en el tema literario y $M=37.07$ en el de biología), mientras que la menor ejecución se presentó en el nivel sustitutivo referencial ( $M=12.22$ y $M=16.00$, respectivamente). En todos los niveles funcionales el tópico de biología obtuvo mayores promedios de ejecución que el literario.

La Tabla 2 muestra los puntajes promedio obtenidos por los alumnos ubicados en el rango Medio de 
Comprensión lectora por tema y nivel funcional

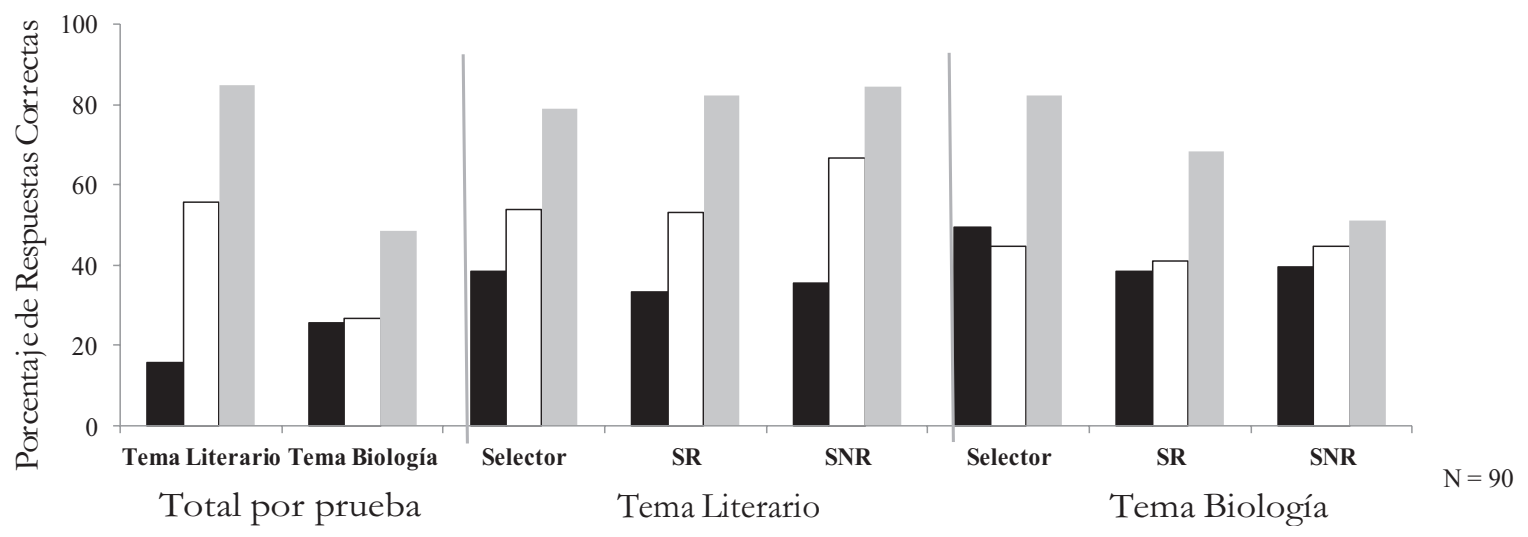

$$
\begin{aligned}
& \text { Rango bajo } \mathrm{N}=27 \\
& \text { Rango medio } \mathrm{N}=44 \\
& \text { Rango alto } \mathrm{N}=19
\end{aligned}
$$

Figura 1. Porcentaje promedio de respuestas correctas obtenidas por los alumnos ubicados en cada rango de calificación en las pruebas de comprensión lectora.

Nota. En el bloque izquierdo se presentan los porcentajes totales obtenidos en cada prueba (tema literario y tema de biología). En los otros bloques se ubican los porcentajes por prueba, en función de cada nivel funcional: Selector, Sustitutivo Referencial (SR) y Sustitutivo No Referencial (SNR).

\begin{tabular}{|c|c|c|c|c|c|c|c|}
\hline \multirow{2}{*}{ Tópico } & \multirow{2}{*}{ Media } & \multirow{2}{*}{$D E$} & \multirow[b]{2}{*}{$t$} & \multirow[b]{2}{*}{$g l$} & \multirow[b]{2}{*}{$p$} & \multicolumn{2}{|c|}{ IC $95 \%$} \\
\hline & & & & & & Inferior & Superior \\
\hline & \multicolumn{7}{|c|}{ Nivel Selector } \\
\hline Literario & 19.70 & 24.95 & -16.72 & 26 & .01 & -90.17 & -70.43 \\
\hline \multirow[t]{2}{*}{ Biología } & 37.07 & 33.86 & -9.65 & 26 & .01 & -76.32 & -49.53 \\
\hline & \multicolumn{7}{|c|}{ Sustitutivo Referencial } \\
\hline Literario & 12.22 & 16.23 & -28.08 & 26 & .01 & -94.20 & -81.35 \\
\hline \multirow[t]{2}{*}{ Biología } & 16.00 & 26.74 & -16.31 & 26 & .01 & -94.58 & -73.42 \\
\hline & \multicolumn{7}{|c|}{ Sustitutivo No Referencial } \\
\hline Literario & 14.74 & 21.32 & -20.77 & 26 & .01 & -93.69 & -76.82 \\
\hline \multirow[t]{2}{*}{ Biología } & 23.41 & 25.90 & -15.36 & 26 & .01 & -86.84 & -66.34 \\
\hline & \multicolumn{7}{|c|}{ Puntaje Total de la prueba } \\
\hline Literario & 15.07 & 13.35 & -33.05 & 26 & .01 & -90.21 & -79.64 \\
\hline Biología & 23.81 & 24.85 & -15.92 & 26 & .01 & -86.02 & -66.35 \\
\hline
\end{tabular}

Tabla 1.

Diferencias en el grupo de desempeño bajo por nivel funcional y puntajes totales de ambas pruebas

Nota. Valor de la prueba 100 
Tabla 2.

Diferencias en el grupo de desempeño medio por nivel funcional y puntajes totales de ambas pruebas

\begin{tabular}{|c|c|c|c|c|c|c|c|}
\hline \multirow{2}{*}{ Tópico } & \multirow{2}{*}{ Media } & \multirow{2}{*}{$D E$} & \multirow{2}{*}{$t$} & \multirow{2}{*}{$g l$} & \multirow{2}{*}{$p$} & \multicolumn{2}{|c|}{ IC $95 \%$} \\
\hline & & & & & & Inferior & Superior \\
\hline & \multicolumn{7}{|c|}{ Nivel Selector } \\
\hline Literario & 51.03 & 26.05 & -10.29 & 29 & .01 & -58.70 & -39.24 \\
\hline \multirow[t]{2}{*}{ Biología } & 35.50 & 30.33 & -11.64 & 29 & .01 & -75.83 & -53.17 \\
\hline & \multicolumn{7}{|c|}{ Sustitutivo Referencial } \\
\hline Literario & 45.53 & 27.12 & -10.99 & 29 & .01 & -64.60 & -44.34 \\
\hline \multirow[t]{2}{*}{ Biología } & 21.17 & 32.24 & -13.38 & 29 & .01 & -90.88 & -66.79 \\
\hline & \multicolumn{7}{|c|}{ Sustitutivo No Referencial } \\
\hline Literario & 55.60 & 29.60 & -8.21 & 29 & .01 & -55.45 & -33.35 \\
\hline \multirow[t]{2}{*}{ Biología } & 31.13 & 36.09 & -10.44 & 29 & .01 & -82.35 & -55.39 \\
\hline & \multicolumn{7}{|c|}{ Puntaje Total de la prueba } \\
\hline Literario & 50.80 & 6.04 & -44.55 & 29 & .01 & -51.46 & -46.94 \\
\hline Biología & 29.13 & 22.17 & -17.50 & 29 & .01 & -79.15 & -62.59 \\
\hline
\end{tabular}

Nota. Valor de la prueba 100.

Tabla 3.

Diferencias en el grupo de desempeño alto por nivel funcional y puntajes totales de ambas pruebas

\begin{tabular}{|c|c|c|c|c|c|c|c|}
\hline \multirow{2}{*}{ Tópico } & \multirow{2}{*}{ Media } & \multirow{2}{*}{$D E$} & \multirow[b]{2}{*}{$t$} & \multirow[b]{2}{*}{$g l$} & \multirow[b]{2}{*}{$p$} & \multicolumn{2}{|c|}{ IC 95\% } \\
\hline & & & & & & Inferior & Superior \\
\hline & \multicolumn{7}{|c|}{ Nivel Selector } \\
\hline Literario & 71.79 & 25.25 & -6.41 & 32 & .01 & -37.17 & -19.26 \\
\hline \multirow[t]{2}{*}{ Biología } & 54.45 & 33.26 & -7.86 & 32 & .01 & -45.54 & -33.75 \\
\hline & \multicolumn{7}{|c|}{ Sustitutivo Referencial } \\
\hline Literario & 76.91 & 21.20 & -6.25 & 32 & .01 & -30.61 & -15.57 \\
\hline \multirow[t]{2}{*}{ Biología } & 26.30 & 32.12 & -13.18 & 32 & .01 & -85.09 & -62.31 \\
\hline & \multicolumn{7}{|c|}{ Sustitutivo No Referencial } \\
\hline Literario & 82.97 & 18.75 & -5.21 & 32 & .01 & -23.68 & -10.38 \\
\hline \multirow[t]{2}{*}{ Biología } & 30.27 & 33.77 & -11.86 & 32 & .01 & -81.70 & -57.75 \\
\hline & \multicolumn{7}{|c|}{ Puntaje Total de la prueba } \\
\hline Literario & 77.33 & 11.31 & -11.50 & 32 & .01 & -26.68 & -18.65 \\
\hline Biología & 37.00 & 23.36 & -15.49 & 32 & .01 & -71.28 & -54.72 \\
\hline
\end{tabular}

Nota. Valor de la prueba 100

calificación, y las diferencias que mostraron entre ambos tópicos, por nivel funcional evaluado y por puntaje global de las pruebas.

A diferencia de lo observado en el grupo Bajo, en el grupo ubicado con un rango Medio de ejecución, el puntaje total obtenido en la prueba sobre tópico literario $(M=50.80)$ fue superior al obtenido en el de biología ( $M$ = 29.13). La comparación por nivel funcional indicó que en el tópico literario la mayor ejecución se presentó en el nivel SNR $(M=55.60)$ y la menor en el nivel SR $(M=$ 
45.53); mientras que en el tópico de biología el puntaje obtenido en el nivel selector fue el mayor $(M=15.07)$ y el menor se ubicó en el SR $(M=21.17)$. Cuando se compararon las respuestas de estos alumnos, tomando el nivel funcional como criterio, sus puntajes en el tema literario fueron mayores que en el de biología, en todos los casos.

La Tabla 3 muestra los puntajes promedio obtenidos por los alumnos ubicados en el rango Alto de calificación, y las diferencias que mostraron entre ambos tópicos, por nivel funcional evaluado y por puntaje global de las pruebas.

$\mathrm{Al}$ igual que el grupo Medio, el grupo ubicado en el rango Alto mostró un puntaje promedio mayor en el total de la prueba del tópico literario $(M=77.33)$, con una diferencia de 36 puntos porcentuales. La mayor ejecución en este tema correspondió al nivel SNR ( $M$ $=82.97)$. En el caso del tópico de biología, el puntaje mayor correspondió al nivel selector $(M=54.45)$. Otra coincidencia entre este grupo y el de rango Medio se observó cuando se compararon las respuestas tomando el nivel funcional como criterio, porque sus puntajes en el tema literario fueron mayores que en el de biología, en todos los casos.

\section{Discusión}

Los datos generales encontrados en el presente estudio pueden ser indicativos de que los alumnos desarrollaron pocas habilidades de comprensión lectora durante su estancia en la escuela primaria y en los primeros dos meses de secundaria, en lo que respecta a los tres niveles de complejidad aquí evaluados. En el caso del tema literario, el 50\% de respuestas correctas como porcentaje promedio indicó deficiencias, pero éstas fueron aún mayores en el caso del tema de biología cuyo desempeño resultó extremadamente bajo (31\% de respuestas correctas). Este primer hallazgo puede relacionarse con los señalamientos de González et al. (2010) y Pérez (2005), en el sentido de que las prácticas didácticas en el aula han descuidado el desarrollo de habilidades de comprensión lectora, y que, cuando se realizan algunos intentos educativos hacia ese objetivo se llevan a cabo dentro de los cursos de Lengua y
Literatura, es decir, utilizando textos literarios, de naturaleza narrativa, dejando de lado textos expositivoargumentativos.

La principal ventaja de las pruebas aquí utilizadas fue la posibilidad de aportar datos relacionados con el nivel funcional de comprensión lectora en que se desempeñaron los alumnos de secundaria en ambos temas. Esto es importante porque el uso del lenguaje implica el dominio de convenciones lingüísticas variadas, siendo necesario conocer el grado de dominio del lenguaje escrito. Dar cuenta de los tres niveles funcionales más complejos de comprensión lectora permitió saber cuándo los estudiantes fueron capaces de ajustarse a los requerimientos de esos tres tipos distintos de interacción con los textos, en particular cuándo pudieron referir y ser referidos a diversos aspectos de la realidad que se presentan en tiempos y espacios distintos a los presentes, lo cual ocurre cuando se dan interacciones referenciales (SR y SNR).

Un análisis más detallado de los datos obtenidos por la muestra total de estudiantes permitió llevar a cabo una comparación de los niveles funcionales de comprensión lectora que se presentaron en cada tópico. Para el caso del tema literario, el mayor porcentaje de respuestas se ubicó en el nivel funcional más complejo, el sustitutivo no referencial; aun cuando no fue un porcentaje precisamente alto, este dato sugiere la posibilidad de que se hayan realizado esfuerzos educativos para lograr que los alumnos desarrollaran habilidades incipientes para el análisis de textos literarios, lo que les permite referir y describir eventos acompañados de algunos juicios argumentados y explicaciones sobre relaciones entre ellos. Aunque también debe considerarse la posibilidad de que, al ser tópicos que los jóvenes pueden relacionar con su vida cotidiana, ellos hayan logrado tales habilidades sin un entrenamiento escolar específico. Cabe señalar que los textos narrativos son de fácil acceso a la población en general y se ajustan más, en estructura, a otras formas de narración de historias (orales, cinematográficas o televisivas).

Contrariamente a lo encontrado en la prueba sobre el tema literario, en el caso del texto de biología los porcentajes de los niveles sustitutivos fueron 
extremadamente bajos. Este hallazgo es preocupante si se consideran los señalamientos de distintos autores que han abordado la enseñanza de las ciencias en secundaria (Izquierdo, 2005; Jiménez, Álvarez \& Lago, 2005), en el sentido de que estos tópicos se abordan fundamentalmente a partir de textos y que dicha enseñanza debe plantearse como objetivos, no sólo la adquisición de conocimientos o datos teóricos y metodológicos por parte de los estudiantes, sino fomentar en ellos el razonamiento y la argumentación. Como señalan Oliveira y Serra (2005), es ampliamente reconocido por los científicos el importante papel que juegan el lenguaje oral y escrito en la educación científica en todos los grados escolares para promover en los alumnos la creatividad y el pensamiento crítico; sin embargo, paradójicamente, éste parece ser infravalorado por los profesores y los autores de libros de ciencias en estudiantes de escuelas secundarias. Planteamientos similares, desde la perspectiva interconductual, han sido realizados por autores como Mares (2008), después de un análisis sistemático de los textos de ciencias naturales y las prácticas didácticas relacionadas con tales temas en escuelas mexicanas.

Los datos obtenidos con los 63 alumnos que se ubicaron en los rangos Medio y Alto de ejecución parecen corroborar la hipótesis que se planteó al inicio del estudio: estos estudiantes mostraron mejores desempeños de comprensión lectora en una prueba basada en un tópico literario, que en una relativa a un tópico de biología.

Los datos correspondientes a los 27 participantes ubicados en el rango Bajo de calificación llaman la atención porque mostraron un patrón de respuestas completamente distinto al de los estudiantes situados en los otros dos rangos. Además de que el desempeño de estos alumnos fue ligeramente mayor en el tema de biología, se evidenció un predominio del nivel selector en ambos tópicos, con porcentajes de ejecución extremadamente bajos en los niveles sustitutivos del tema literario. De hecho, su desempeño en dicho tópico no alcanzó el 20\% de respuestas correctas. Estos datos pueden indicar que los lectores menos hábiles se comportan en niveles funcionales de menor complejidad, independientemente del tema de lectura del que se trate, porque no están acostumbrados a relacionar los temas que leen con su vida cotidiana $y$ menos aún a reflexionar sobre la importancia práctica, conceptual o científica de los tópicos que se abordan en sus textos escolares. Aunque esto ha de corroborarse en estudios dirigidos expresamente a investigar qué tipo de asociaciones, análisis y reflexiones se promueven en el aula, y cuáles de estas estrategias son utilizadas por los alumnos al leer y estudiar textos de diversas materias, dentro y fuera del aula.

Lo que sí es posible afirmar, utilizando los términos derivados del interconductismo, es que los participantes del presente estudio especialmente los que mostraron desempeños bajos y medios no cumplen con los criterios señalados por autores como Fuentes (2010) e Irigoyen et al. (2007) para ser considerados como lectores competentes, dado que muestran fallas para leer textualmente y para responder preguntas de comprensión en los diversos niveles evaluados, en especial para el tópico de biología. Esta comprensión limitada, como explica Mares (2008) puede llevar a que el alumno tenga problemas para generalizar o para aplicar el conocimiento, es decir, que limite la transferencia de lo aprendido.

La presente investigación tiene limitaciones, como el hecho de presentar datos de alumnos de una sola escuela (pública) y que las pruebas utilizadas sólo cuentan con validez de contenido. Por ello, cabe aclarar que los datos aquí reportados no pueden ser generalizados a toda la población escolar mexicana de secundaria, aunque sí muestran evidencia de la necesidad de evaluar la comprensión lectora considerando distintos niveles de complejidad, y de que tales evaluaciones se encaucen a conocer las diferencias en los desempeños de los estudiantes en distintos temas.

La presente investigación también aporta algunas directrices sobre cómo encaminar el desarrollo de habilidades de lectura en los alumnos de educación básica, al evidenciar la necesidad de programar la instrucción de la comprensión lectora incrementando sucesivamente el grado de complejidad de los intercambios lingüísticos en el aula, para favorecer 
interacciones sustitutivas con los textos académicos. Con apoyo en un marco teórico como el que aquí se utiliza, futuras investigaciones han de dirigirse al cumplimiento pleno de tal objetivo.

La investigación en este rubro puede tener especial utilidad para poblaciones estudiantiles desfavorecidas porque, como se desprende de las evaluaciones realizadas por el Instituto Nacional para la Evaluación Educativa, los desempeños de los alumnos se ven afectados directamente por aspectos como el contexto social, cultural y económico del que provienen. A partir de ello, Sánchez y Andrade (2009) concluyen que el sistema educativo mexicano no ha logrado la implantación de programas que puedan remontar las deficiencias que muestran los estudiantes provenientes de estratos socioculturales bajos, lo cual implica que el propio Estado preserve las condiciones de desigualdad en la distribución de oportunidades de formación escolar, con un fuerte impacto en la equidad, como dimensión de la calidad educativa.

Atender las necesidades educativas de dicha población estudiantil debe ser un objetivo prioritario de las políticas públicas, pero también de la investigación psicológica educativa.

\section{Referencias}

Arroyo, R., Morales, G., Silva, H., Camacho, I., Canales, C., \& Carpio, C. (2008). Análisis funcional del conocimiento previo: sus efectos sobre el ajuste lector. Acta Colombiana de Psicología, 11(2), 55-64.

Arroyo, R., Morales, G., Silva, H., Canales, C., \& Carpio, C. (2011). Evaluación de habilidades lectoras en la educación superior. En J. Irigoyen, K., Acuña \& M. Jiménez (Coord.), Evaluación de desempeños académicos (pp. 97-106). México: Universidad de Sonora.

Backhoff, E., Sánchez, A., Peón, M., \& Andrade, E. (2010). Comprensión lectora y habilidades matemáticas de estudiantes de educación básica en México: 2000-2005. Revista Electrónica de Investigación Educativa, 12 (1). Recuperado de: http://redie.uabc. $\mathrm{mx} /$ vol12no1/contenido-backhoffsanchez.html
Bazán, A., Castañeda S., Macotela S., \& Valenzuela, M. (2004). Evaluación del desempeño en lectura y escritura. Aportes empíricos a la noción de componentes lingüísticos en el cuarto grado de primaria. Revista Mexicana de Investigación Educativa, 9(23), 841-861.

Fuentes, M. T. (2010). La comprensión lectora: revisión teórica desde la perspectiva cognoscitiva, conductual e interconductual. En T. Fuentes, J. Irigoyen, \& G. Mares (Eds.), Tendencias en psicología y educación. Revisiones temáticas. Volumen 1 (pp. 128-166). México: Red Mexicana de Investigación en Psicología Educativa.

González, J., Barba, J., \& González, A. (2010). La comprensión lectora en educación secundaria. Revista Iberoamericana de Educación, 53(6), 1-11. Recuperado de: http://www.rieoei.org/expe/3225Gonzalez.pdf

Guevara, Y. (2006). Análisis interconductual de algunos elementos que constituyen la enseñanza básica. Revista Mexicana de Investigación Educativa, 11(30), 1037-1064.

Ibáñez, C., \& Ribes, E. (2001). Un análisis interconductual de los procesos educativos. Revista Mexicana de Psicología, 18(3), 359-371.

Irigoyen, J., Acuña, K., \& Jiménez, M. (2011). Interacciones didácticas en educación superior. Algunas consideraciones sobre la evaluación de desempeños. En J. Irigoyen, K. Acuña, \& M. Jiménez (Coords.), Evaluación de desempeños académicos (pp. 7396). México: Universidad de Sonora.

Irigoyen, J., Jiménez, M., \& Acuña, K. (2007). Evaluación de la comprensión lectora en el aprendizaje de la ciencia psicológica. En J. Irigoyen, M. Jiménez, \& K. Acuña (Eds.), Enseñanza, aprendizaje y evaluación. Una aproximación a la pedagogía de las ciencias (pp. 213-246). México: Universidad de Sonora.

Izquierdo, M. (2005). Estructuras retóricas en los libros de ciencias. Revista de Investigación e Innovación Educativa, 36, 11-34. Recuperado de: http://www.uam.es/iuce/ iuceweb/publicaciones/tarbiya/tarbiya.htm

Jiménez, P., Álvarez, V., \& Lago, J. (2005). La argumentación en los libros de texto de ciencias. Revista de Investigación e Innovación Educativa, 36, 35-58. Recuperado de: http://www. uam.es/iuce/iuceweb/publicaciones/tarbiya/tarbiya.htm 
Llorens, C., Gil, L., Vidal-Abarca, E., Martínez, T., Mañá, A., \& Gilabert, R. (2011). Prueba de competencia lectora para educación secundaria (CompLEC). Psicothema, 23(4), 808-817.

Mares, G. (2008). Promoción de competencias a través de textos para la enseñanza de las ciencias naturales. En G. Mares (Coord.), Diseño pedagógico de textos: diversos enfoques (pp. 171-200). México: Universidad Nacional Autónoma de México.

Mares, G., Rueda, E., Rivas, O., \& Rocha, H. (2009). Maneras de leer que promueven el aprendizaje y su transferencia. México: Universidad Nacional Autónoma de México.

Oliveira, M., \& Serra, P. (2005). La creatividad, el pensamiento crítico y los textos de ciencias. Revista de
Investigación e Innovación Educativa, 36, 59-80. Recuperado de http://www.uam.es/iuce/iuceweb/publicaciones/ tarbiya/tarbiya.htm

Pérez, M. J. (2005). Evaluación de la comprensión lectora: Dificultades y limitaciones. Revista de Educación, Número extraordinario, 121-138. Recuperado de: http://www.revistaeducacion. mec.es/re2005/re2005_10.pdf

Ribes, E., \& López, F. (1985). Teoría de la conducta. Un análisis de campo y paramétrico. México: Trillas.

Sánchez, A., \& Andrade, E. (2009). El aprendizaje en tercero de secundaria en México. Informe sobre los resultados del Excale 09, aplicación 2008 Español, Matemáticas, Biología y Formación cívica y ética. México: Instituto Nacional de Evaluación Educativa. 
